\title{
Theoretical study for Coronene and Coronene-Al, B, C, Ga, In and Coronene-O interactions by using Density \\ Functional theory \\ https://doi.org/10.32792/utq/uti/vol14/4/6
}

\section{Abbas Sh. Alwan, Sadiq Kh. Ajeel, Mohammed L. Jabbar \\ Physics Department, College of science, Thi-Qar \\ University, Iraq}

Emails: abbasshwaa_ph@sci.utq.edu.iq

abbasalwan@hotmail.com

\section{Abstract}

Molecular structure geometry have been investigated for Coronene and Coronene-Al, B, C, Ga, In and Coronene-O by using Gaussian software at basis set 3-21G, B3LYP level, density functional theory (DFT). Adding the atoms $\mathrm{Al}, \mathrm{B}, \mathrm{C}, \mathrm{Ga}$, In or Oxygen to Coronene change electrons density distribution. Study of the magnetic characterstics shows that some systems like Coronene-Carbon and Coronene-oxygen has the antiferromagnetic charactersti, this type of magnetic properties stands for open shel system which has two type of orbital $\alpha$ and $\beta$ orbitals. Individual atoms calculations was achieved in order to make a comparison between Coronene and the atoms to demonstrate which of them will behave as a donor or an acceptor. $\mathrm{E}_{\text {HOMO }}, \mathrm{E}_{\mathrm{SOMO}}, \mathrm{E}_{\mathrm{LUMO}}$, total energy electronegativity and electrophilicity were evaluated for all paradigms under the study. Using DFT to study the symmetry shows that there are two types of point group symmetries $\mathrm{C}_{6 \mathrm{~h}} / \mathrm{C}_{1}$ and $\mathrm{Cs} / \mathrm{C}_{1}$. Coronene-Corbon posseses the highest dipole moment value among the samples, it has the value (5.5873 Debye), dipole moment credit is very important to give sight about the internal structure of substances. Calculations of chemical potential shows clearly $\mathrm{Al}, \mathrm{Ga}$ and In behave as donors, while $\mathrm{B}, \mathrm{C}$ and $\mathrm{O}$ behave as acceptors. Also binding energy study exhibits that Coronene is going to physisorbe on the surface of the atoms $\mathrm{B}, \mathrm{C}$ and $\mathrm{O}$.

Keywords: DFT, Contours, Symmetry, Dipole moment, Binding energy.

\section{Introduction}


One can investigate the electronic structure of particular atoms, molecules, nanoclusters, nanoribons, nanoballs and substances by using quantum mechanics methods such as Hartree-Fock approximation, post Hartree-Fock methods and density functional theory [1]. Density functional approaches form the basics of a variety and very active sciences nowadays computational atomic, molecular, nuclear and even solid state physics [2]. Density functional theory treats with the systems that have many particles throughout the electron density [3]. Electron density is the likelihood of determining existence places of the electrons at certain space[4]. Density functional theory suppose the electron density instead of the wave function, and also it assumes the electron density only depends on three spatial coordinates without regarding how the electrons are existed in the systems, while the wave function approximation considers $3 \mathrm{~N}$ variables, three spatial coordinates, one coordinate for spin by assumption the nuclei location is fixed [5]. Density functional theory seek on ground state energy, ane also say it seach on approximation solution to schrodinger equation of many electrons system [6]. In DFT many electrons system can described by functionals [7]. DFT calculation agree tremendously with the practical results. Gaussian orbitals in density functional approximations can expressed as a Gaussian function vary exponentially with the square of the position of the electrons [8]. Using B3LYP basis set in density functional theory gives precisely consequences than other approaches [9]. Exchange correlation functionals are from the important instruments in the computations of density functional theory [10]. Total atom charge is calculated depending on Mullkin method when on use density functional theory for studying properties of many electrons system [11]. Computations of density functional approximations take in account inner shell electrons, inner valence electrons and outer valence electrons [12].

\section{Results and Discussion}

\section{Molecular Geometry}

Molecular structure point out arrangement of atoms in the molecule, it denotes to positions of atoms in the substance[13]. Molecular structure maps clarify the optimized parameters, bond lengths, bond angles and dihedral angles [14]. Optimization of Coronene and Coronene-Al, Coronene-B, Coronene- $\mathrm{C}$, Coronene-Ga, Coronene-In and Coronene-O interactions have been carried out with Gaussian 09 software package. 


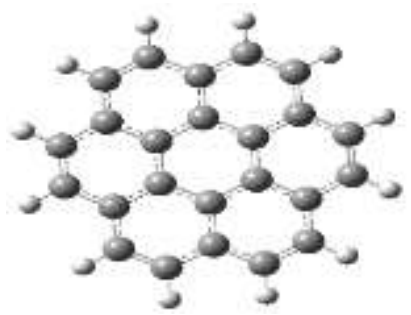

Figure (1)

Molecular structure of Coronene

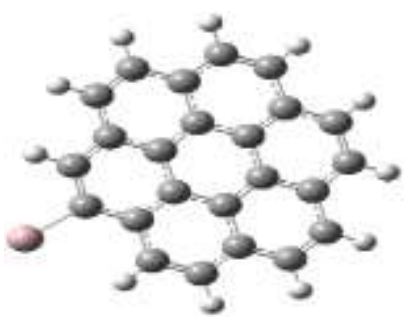

Coronene-Al

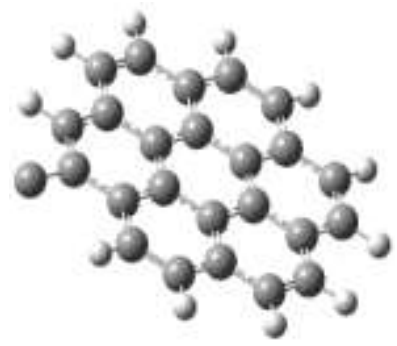

Coronene- $\mathrm{C}$

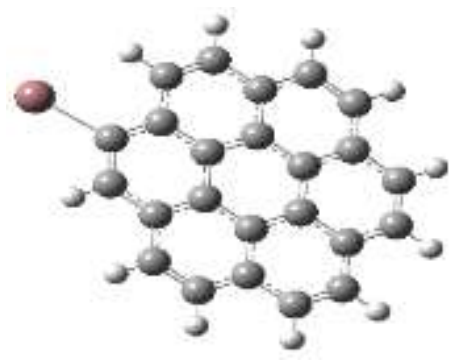

Coronene-In

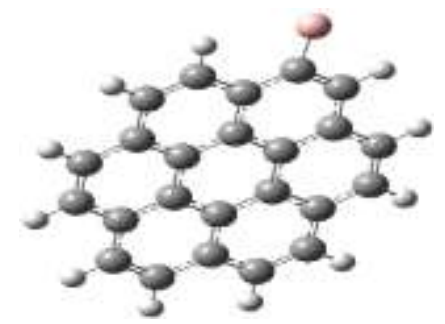

Coronene-B

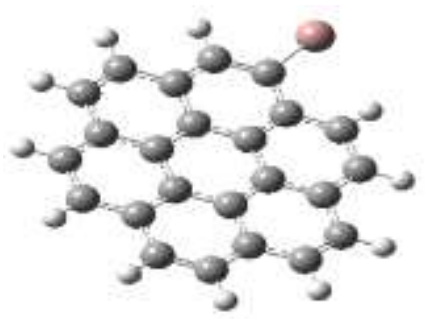

Coronene-Ga

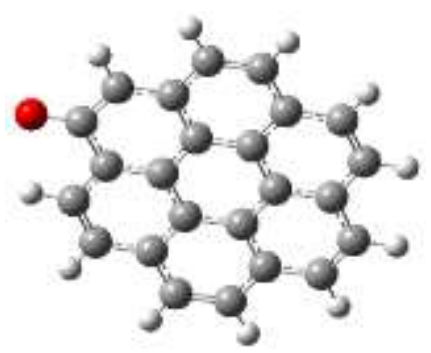

Coronene-O

Figure (2) 


\section{Molecular structure of Coronene-Al, B, C, Ga, In and Coronene-O}

From Figure (1) one can say the molecular structure of Coronene and Coronene-Al, Coronene-B, Coronene-C, Coronene-Ga, Coronene-In and Coronene-O have been gained throughout the geometry optimization procedure that search on stationary points on the potential surfaces. Molecular structure describe the topological properties, bond angles among any three atoms and dihedral angles among any four atoms. In order to get the optimized molecular structure the geometry optimization seek on the lowest energy.

\section{Contours}

One can express the electrons density throughout the contours [15]. Contours have been implemented at electrostatic potential (ESP). Contour maps explain mechanism of the reaction throughout determination the active sites in the molecule. Contour maps is very useful in the study of charge exchange and energy transfer procedures, also the contours maps yield the spatial distribution with the surface [16]. Electrostatic potential maps have been investigated for Coronene and Coronene - Al, B, C, Ga, In, $\mathrm{O}$ interactions.

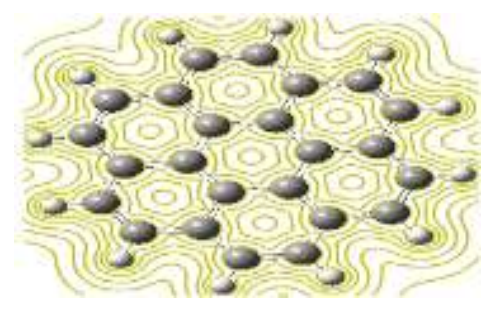

Figure(3)

Contours for Coronene 


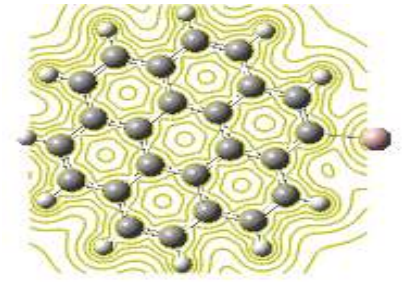

Coronene-Al

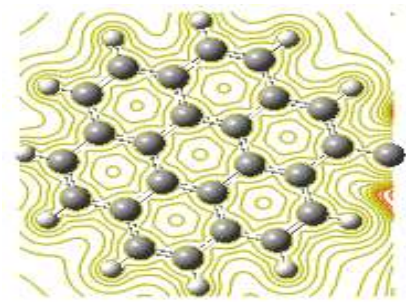

Coronene- $\mathrm{C}$

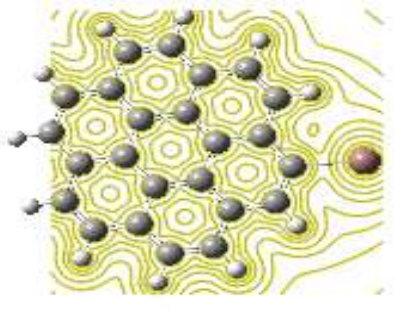

Coronene-In

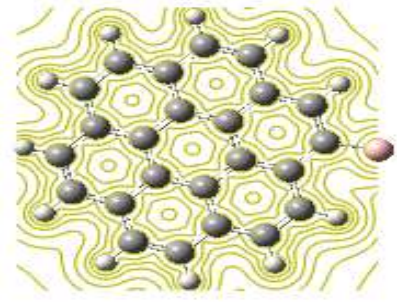

Coronene-B

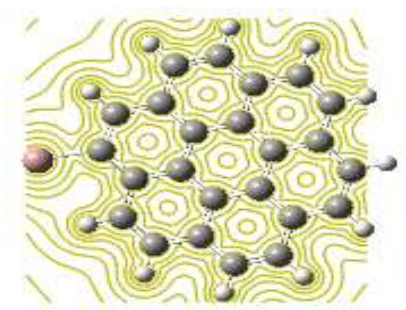

Coronene-Ga

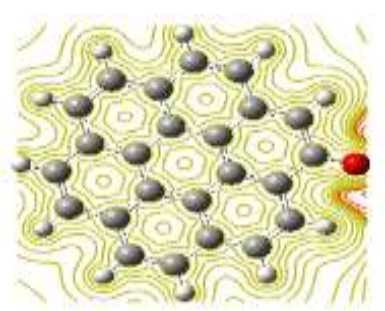

Coronene-O

Figure(4)

\section{Contours of Coronene-Al, B, C, Ga, In and Coronene-O}

In Figures (3) and (4) one can remark electronical density contours, contour maps show clarity charge density distribution around atoms. Adding atoms of Aluminume, Boron, Carbon, Gallium, Indium and Oxygen to Coronene make distortion in contour maps, this refers to the interaction of those atoms with Coronene surface. Also contour diagrams 
give informations about interaction behavior and the probability to come about. Wave packet propagation of electrons tremendously associated with contour schemes. Charge distribution around Oxygen atom is the maximum among all atoms

that adding to Coronene, also one can see contour distortion as a result to addition of Carbon atom to Coronene is higher than all of Aluminume, Boron, Gallium and Indium, that is to say charge distribution about the other atoms (Aluminume, Boron, Gallium and Indium) except Oxygen.

\section{Magnetic Properties}

Magnet is very important in many useful devices, such as electric generators, electric transformers and also computers. Solid substances are divided according to response to the magnet into many types:paramagnetic, diamagnetic, ferromagnetic, anti-ferromagnetic and ferrimagnetic [17]. Magnetic credits for Coronene and Coronene- Al, B, $\mathrm{C}, \mathrm{Ga}, \mathrm{In}, \mathrm{O}$ were investigated through geometrical optimization process.

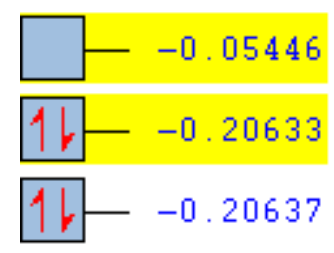

Figure(5)

Magnetic properties for Coronene 


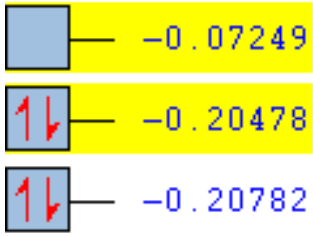

Coronene-Al

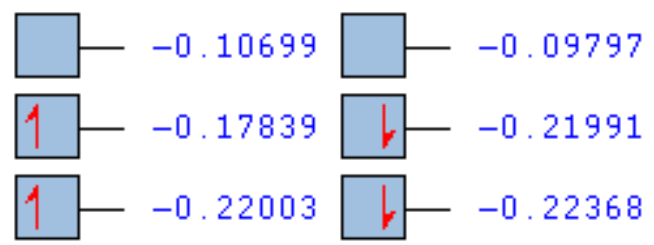

Coronene-C

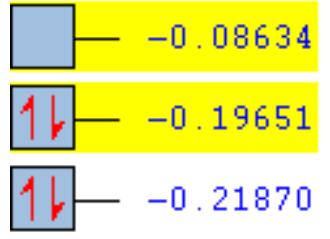

Coronene-B

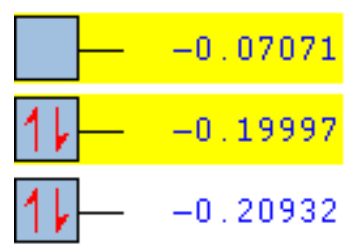

Coronene-Ga

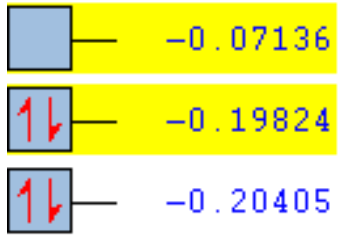

Coronene-In

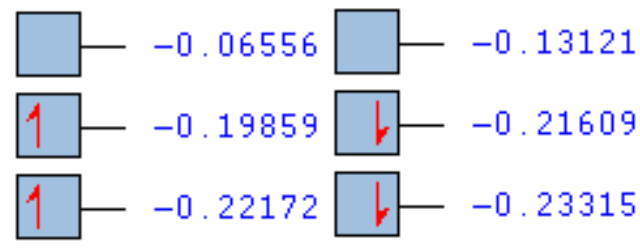

Coronene-O

Figure(6)

Magnetic properties for Coronene and Coronene-Al, B, C, Ga, In, O

One can see in figure(5) and Figure(6) the difference in magnetic properties for samples under the study spin property demonstrate if the sample is closed or open system. Coronene, Coronene-Al, Coronene-B, Coronene-Ga and Coronene-Al are of the type closed because the occupied ultimate orbital is perfectly filled, but the occupies final orbital in Coronene- $\mathrm{C}$ and Coronene-O is partially filled with electrons, ie. those systems open systems. Open system describe the anti-ferromagnetic characterstic, Coronene-C and Coronene- $\mathrm{O}$ anti-ferromagnetic systems. 


\section{Individual atoms Calculations}

Computations of individual atoms is very important for discussion some properties materials. Some of these properties are total energy, electronegativity and electrophilicity as in table (1).

Table (1) shows individual atoms(Al, B, C, G, In and O) calculations.

\begin{tabular}{|c|c|c|c|c|c|}
\hline atom & $\begin{array}{c}\text { HOMO } \\
(\mathbf{e V})\end{array}$ & $\begin{array}{c}\text { LUMO } \\
(\mathbf{e V})\end{array}$ & $\begin{array}{c}\text { Total energy } \\
(\mathbf{e V})\end{array}$ & Electronegativity & Electrophilicity \\
\hline $\mathrm{Al}$ & -3.5484561 & 2.0766672 & -6558.802772 & 0.73589445 & 0.096271782 \\
\hline $\mathrm{B}$ & -4.900521 & -2.4361113 & -667.1519136 & 3.66831615 & 5.460351571 \\
\hline $\mathrm{C}$ & -5.153574 & -3.4834242 & -1022.359854 & 4.3184991 & 11.16632441 \\
\hline $\mathrm{Ga}$ & -3.3242457 & -1.9308216 & -52117.89136 & 2.62753365 & 4.954653132 \\
\hline $\mathrm{In}$ & -3.2262897 & -2.0129958 & -155575.2965 & 2.61964275 & 5.656113607 \\
\hline $\mathrm{O}$ & -8.6565894 & -6.4993806 & -2028.639308 & 7.577985 & 26.62044428 \\
\hline
\end{tabular}

\section{Symmetry}

In general there are seven elements of the symmetries that molecular systems own, those seven elements are identity, proper axis, horizontal plane, vertical plane, dihedral plane, improper axis and inversion center or center of symmetry [18]. All element contains symmetry operation. Point group symmetries in the present study were obtained for Coronene and Coronene and Coronene-Al, B, C, Ga, In, O through the geometry optimization procedure of the studying systems.

Table (2) shows the point group symmetries of Coronene and Coronene and Coronene-Al, B, C, Ga, In, $\mathrm{O}$.

\begin{tabular}{|l|c|}
\hline \multicolumn{1}{|c|}{ System } & Symmetry \\
\hline Coronene & $\mathrm{C} 6 \mathrm{~h} / \mathrm{C} 1$ \\
\hline Coronene-Al & $\mathrm{Cs} / \mathrm{C} 1$ \\
\hline Coronene-B & $\mathrm{Cs} / \mathrm{C} 1$ \\
\hline Coronene-C & $\mathrm{Cs} / \mathrm{C} 1$ \\
\hline Coronene-Ga & $\mathrm{Cs} / \mathrm{C} 1$ \\
\hline Coronene-In & $\mathrm{Cs} / \mathrm{C} 1$ \\
\hline Coronene-O & $\mathrm{Cs} / \mathrm{C} 1$ \\
\hline
\end{tabular}

In Table (2) one can show that Coronene has the symmetry $\mathrm{C}_{6 \mathrm{~h}} / \mathrm{C}_{1}$, one can say the rotation in Coronene obtains by $60^{\circ}$ since Coronene of the kind of that has rotation repeats the same molecule by (360/n) degrees, 
also, Coronene has horizontal mirror plane (h). All other systems (Coronene, Coronene-Al, Coronene-B, Coronene-Ga and Coronene-Al Coronene- $\mathrm{C}$ and Coronene-O) have two elements identity and mirror plane, Also all those systems repeat themselves throughout $360^{\circ}$. Symmetry is very important credit, it gives informations obout the molecular structures without calculations.

\section{Dipole moment}

Dipole moment represents charge multiplying by the displacement between two signal different charges one is positive and the other is negative. Dipole moment illustrates in hetronuclear molecules. It can be described by the following relation[19]

$$
P=q r
$$

Table (3) shows the dipole moments for Coronene and Coronene - Al, B, C, Ga, In, O interactions.

\begin{tabular}{|l|c|}
\hline \multicolumn{1}{|c|}{ System } & Dipole moment (Debye) \\
\hline Coronene & 0.0000 \\
\hline Coronene-Al & 1.4223 \\
\hline Coronene-B & 5.0211 \\
\hline Coronene-C & 5.5873 \\
\hline Coronene-Ga & 1.9236 \\
\hline Coronene-In & 0.2504 \\
\hline Coronene-O & 4.5746 \\
\hline
\end{tabular}

From Table (3) on can visualize that Coronene has zero value of the dipole moment although it has two type of atoms Carbon and Hydrogen, this occurs because of many factors such as the molecular structure and symmetry. Adding Boron atom to Coronene makes it has value of dipole moment equal to (5.0211 Debye) because the system Coronene-Boron Hetronuclear, Also this happen because of the hybridization procedure. So Coronene-Aluminume, Coronene-Carbon, Coronene-Oxygen and Coronene-Gallium Hetronuclear, therefore they have nonzero values of dipole moment. Coronene-Indium has semi-zero value of the dipole moment although it is hetronuclear system this also takes place because of many factors like the molecular structure, symmetry and hybridization. 


\section{Donor Acceptor property}

Electronegativity show trend of an atom for attracting a bonding pair of electrons. It is a property show tendency of the electrons to escape from equilibrium system, this system perhaps atoms or molecules system. The mathematical description of the electronegativity as follows[20]

$$
\mu=\left(\frac{\partial E}{\partial N}\right)_{V(r)} \approx-^{\chi}
$$

Electrophilicity is the property that measure the energy lowering which result from maximum electron flow between donor and acceptor. The following equation show how the electrophilicity is calculated [21]

$$
\omega=\frac{\mu^{2}}{2 \eta}
$$

Table (4) shows the electronegativities and electrophilicities for Coronene and Coronene - Al, B, C, Ga, In, O interactions.

\begin{tabular}{|l|c|c|}
\hline \multicolumn{1}{|c|}{ System } & Electronegativity & Electrophilicity \\
\hline Coronene & 3.54804795 & 1.291590487 \\
\hline Coronene-Al & 3.77225835 & 1.551508697 \\
\hline Coronene-B & 3.84817425 & 1.774579507 \\
\hline Coronene-C & 3.8825949 & 2.217892895 \\
\hline Coronene-Ga & 3.6826014 & 1.513845256 \\
\hline Coronene-In & 3.667908 & 1.520773863 \\
\hline Coronene-O & 3.59376075 & 1.431294708 \\
\hline
\end{tabular}

According to Table (4) one can classify Coronene and the adding atoms if it behave as a donor or an acceptor. With consideration to this table in Coronene-Al, Coronene-Ga and Coronene-In, the adding atoms (Al, Ga and In) behave as donors while Coronene became an acceptor, electrons of $\mathrm{Al}, \mathrm{Ga}$ and $\mathrm{In}$ atoms adsorbed on Coronene surface. While in Coronene- $\mathrm{B}$, Coronene- $\mathrm{C}$ and Coronene- $\mathrm{O}$ come about the contrary, Coronene behaves as a donor, but Boron atom, Carbon atom and Oxygen atom become acceptors, electrons of coronene will physisorbe on $\mathrm{B}, \mathrm{C}$ and $\mathrm{O}$ atoms. 


\section{Binding energy}

In general binding energy denotes to the work done against the electric attraction. Sometimes it is called the separation energy, because it is the energy required to decompose atom, molecule or nucleus into its constituent particles [22]. Mathematically it has the formula [23]

$$
E_{B}=n E(X)+m E(Y)-E\left(X_{n} Y_{m}\right)
$$

$E(X)$ is the energy of the molecule $\mathrm{X}, E(Y)$ is the energy of the molecule $\mathrm{Y}, E\left(X_{n} Y_{m}\right)$ is the energy of the molecule $X_{n} Y_{m}, n$ is number of atoms for the molecule $\mathrm{X}, m$ is Number of atoms for the molecule Y.

Table (5) shows the binding energies for Coronene and Coronene Al, B, C, Ga, In, O.

\begin{tabular}{|l|c|}
\hline \multicolumn{1}{|c|}{ System } & Binding Energy(eV) \\
\hline Coronene-Al & -481936.5177 \\
\hline Coronene-B & 17486.05166 \\
\hline Coronene-C & 26796.05177 \\
\hline Coronene-Ga & 1366009.933 \\
\hline Coronene-In & 4077628.521 \\
\hline Coronene-O & 53170.63626 \\
\hline
\end{tabular}

Table (5) exhibits the binding energy of the ground state, binding energies shows how (Al, Ga and In) physisorbs on Coronene surface, so visualize that Coronene atoms can adsorbed on $\mathrm{B}, \mathrm{C}$ and $\mathrm{O}$ atoms surfaces. Binding energies between Coronene surface and ( $\mathrm{Al}, \mathrm{Ga}, \mathrm{In}, \mathrm{B}, \mathrm{C}$ and $\mathrm{O})$ indicates to that the interaction between Coronene surface and those atoms takes place at certain distances represents the bond lengthes. The binding energies refer to a physical interaction between ( $\mathrm{Al}, \mathrm{Ga}, \mathrm{In}, \mathrm{B}, \mathrm{C}$ and $\mathrm{O}$ ) and Coronene surface. The linear combination of the atomic orbitals between Coronene and ( $\mathrm{Al}, \mathrm{Ga}, \mathrm{In}, \mathrm{B}, \mathrm{C}$ and $\mathrm{O}$ ) forms the binding energies in Coronene-Al, Coronene-Ga, Coronene-In, Coronene-B, Coronene- $\mathrm{C}$ and Coronene Oxygen. 


\section{Conclusions}

Molecular geometry maps clarify that the distances (bond angles), bond angles and dihedral angles between Coronene surface and the adding individual atoms are different, that is to say charge transfer between theatoms and Coronene surface obtain at different optimized parameters. Contour maps diagrams demonstrate the distortion in the wave function of electrons, electrons density distributions and electrostatic potential. Electrons density distribution in the samples Coronene-C and Coronene$\mathrm{O}$ is clear tremendously largerger than the other samples through Contour diagrams. Only alpha orbitals result in Coronene and Coronene- $\mathrm{Al}, \mathrm{Ga}$, In, B, but alpha and beta orbitals consequent in the samples Coronene-C and Coronene- $\mathrm{O}$, those two paradigms has the $\mathrm{E}_{\mathrm{SOMO} O}$ and $\mathrm{E}_{\mathrm{LUMO}}$, one can assert those two patterns have antiferromagnetic characterstics. It is very clear that adding any atom to Coronene in this study make the symmetry properties different. Coronene-In has the minimum value of dipole moment, but Coronene-C hasthe maximum valueof dipole moment. Coronene behave as a donor in the paradigms Coronene-Al, Ga, In, but it behaves as an acceptor in Coronene-B, C, O through charge transfer procedures. Also computations of electronegativity characterstic shows clearly $\mathrm{Al}, \mathrm{Ga}$ and In behave as donors, while $\mathrm{B}, \mathrm{C}$ and $\mathrm{O}$ behave as acceptors. Coronene-Al has negative value of the binding energy because of the nature of bonding which gets throughout geometry optimization procedure.

\section{Acknowledgements}

Our thankful for Prof. Dr. Hamid I. Abboud, Prof. Dr. Falah H. Hannon and Asst. prof Fouad N. Ajeel.

\section{References}

[1] Assadi, M.H.N, et al. Theoretical study on copper's energetic and magnetism in $\mathrm{TiO} 2$ polymorphs. (2013).

[2] Van Maurik, Tanjia, Gdanitz, Robert J. Acritical note on density functional theory studies on rare-gas dimmers. Journal of Chemical Physics 116 (22): 9620-9623. (2002).

[3] ] Zimmerli., Urs., Parrinello., Michele., Koumoutsakos., Petros., Dispersion corrections to density functionals for water aromatic interactions, Journal of Chemical Physics 120 (6): 2693-2699. (2004). [4] Parr, Robert G., Yang, Weitao, Density-Functional Theory of Atoms and Molecules, Oxford University Press, (1994). 
[5] C.J. Carmer, Essential of Computational Chemistry, Chichester, John Wiley and Sons, Ltd, 154-168. (2002).

[6] C.J. Carmer, Essential of Computational Chemistry, Chichester: John Wiley and Sons, Ltd, (2002), 154-168.

[7] Wolfram Koch, Max C. Holthausen, A Chemist's Guide to Density Functional Theory, $2^{\text {nd }}$ Edition, Wiley-VCH Verlag GmbH, Germany, (2001).

[8] G. Robert, D.Yang, Weitao, Density-Functional Theory of Atoms and Molecules, Oxford University Press, (1994).

[9] C. Lee, W. Yang, R.G. Parr, Development of the Colle-Salvetti correlation-energy formula into a functional of the electron density, Phys. Rev. B 37, 785, (1988).

[10] Jack Simons, An Introduction to Theoretical Chemistry, Salt Lake City, Utah, University of Utah, Chemistry Department, (2000). [11] Ali A. M., Investigations Of Some Antioxidant Materials By Using Density Functional And Semiempirical Theories, P.hD. Thesis, University of Basrah, College of Science, Department of Physics, (2009).

[12] Jensen, Frank, Introduction to computational chemistry. JohnWiley and Sons. Pp. 68-77. (1999).

[13] M. J. Frisch, G.W. Trucks, H. B. Schlegel, G. E. Scuseria, M. A. Robb, J. R. Cheeseman, G.Scalmani, V. Barone, B. Mennucci, G. A. Petersson, H. Nakatsuji, M. Caricato, X. Li, H. P. Hartchain, A. F. Izmaylov, J. Bloino, G. Zheng, J. L. Sonnenberg, M. Hada, M. Ehara, K. Toyota, R. Fukuda, J. Hasegawa, M. Ishida, T.Nakajima, Y. Honda, O. Kitao, H. Nakai, T. Vreven, J. A. Montgomery, Jr., J. E. Peralta, F. Ogliaro, M. Bearpark, J. J. Heyd, E. Brothers, K. N. Kudin, V. N. Staroverov, R. Kobayashi, J. Normand, K. Raghavachari, A. Rendell, J. C. Burant, S. S. Iyengar, J. Tomasi, M. Cossi, N. Rega, J. M. Millam, M. Klene, J. E. Knox, J. B. Cross, V. Bakken, C. Adamo, J.Jaramillo, R. Gomperts, R. E. Stratmann, O. Yazyev, A.J. Austin, R. Cammi, C. Pomelli, J. W. Ochterski, R. L. Martin, K. Morokuma, V.G. Zakrzewski, G. A. Voth, P. Salvador, J.J. Dannenberg, S. Dapprich, A. D. Daniels, O.Farkas, J. B. Foresman, J. V. Ortiz, J. Cioslowski and D. J. Fox, "Gaussian 09", revision A. 1, Gaussian, Inc., Wallingford CT, (2009). 
[14] H. I. Abood., Density Functional Theory Calculations of Di-amino naphthalene, Journal of Babylon university, Pure snd Applied Science, 3(22). (2014).

[15] Frisch M. J., G. W. Trucks, H. B. Schlegel et al., Gaussian 09, Revision A.02, Gaussian, Inc., PA, Wallingford CT. (2009).

[16] A. G. Borisov, A. K. Kazansky, and J. P. Gauyacq, Phys.Rev. B,59, 1610935 (1999).

[17] Charles Kittel., Introduction to solid state physics, (1991).

[18] A.M. Lesk, Introduction to symmetry and group theory for chemists, Kluwer Academic Publishes, London, (2004).

[19] Mc Grow-Hill, Fundamentals of molecular spectroscopy, (1972).

[20] Szabo, A.; Ostlund, N. S. Modern Quantum Chemistry:Introduction to Advanced Electronic Structure Theory, Dover Publications, Inc.:New York, (1989).

[21] P. Udhayakala, T. V. Rajendiran, S. Seshadri, and S. Gunasekaran, Quantum chemical vibrational study, molecular property and HOMOLUMO energies of 3-bromoacetophenone for Pharmaceutical application, J. Chem. Pharm. Res., 3, 610-625, (2011).

[22] R.G. Pearson, Recent advances in the concept of hard and soft acids and bases, Journal of

Chem. Edu., 64(1987), 561-567.

[23] Vipin Kumar, Esha V. Shah, Debesh R. Roy, Physica E, Lowdimensional Systems and Nanostructures, (2015). 